\title{
THE DISTRIBUTION OF THE LOGARITHM IN AN ORTHOGONAL AND A SYMPLECTIC FAMILY OF $L$-FUNCTIONS
}

\author{
BOB HOUGH
}

\begin{abstract}
We consider the logarithm of the central value $\log L(1 / 2)$ in the orthogonal family $L(s, f)_{f \in H_{k}}$ where $H_{k}$ is the set of weight $k$ Hecke-eigen cusp form for $S L_{2}(\mathbb{Z})$, and in the symplectic family $L\left(s, \chi_{8 d}\right)_{d \asymp D}$ where $\chi_{8 d}$ is the real character associated to fundamental discriminant $8 d$. Unconditionally, we prove that the two distributions are asymptotically bounded above by Gaussian distributions, in the first case of mean $-1 / 2 \log \log k$ and variance $\log \log k$, and in the second case of mean $1 / 2 \log \log D$ and variance $\log \log D$. Assuming both the Riemann and Zero Density Hypotheses in these families we obtain the full normal law in both families, confirming a conjecture of Keating and Snaith.
\end{abstract}

\section{INTRODUCTION}

An important problem in analytic number theory is to understand the distribution of values of $L$-functions on the central line $\Re(s)=\frac{1}{2}$. Selberg [13 famously proved that as $t$ varies in large intervals $t \in[T, 2 T]$, the real and imaginary parts of the logarithm of Riemann's zeta function become distributed like independent Gaussian random variables. Since that work, there have been several efforts to extend the result to a more general setting. A few years later, Selberg himself [12] proved that for a fixed value of $t$ the imaginary part of $\log L\left(\frac{1}{2}+i t ; \chi\right)$ becomes normally distributed as $\chi$ varies among Dirichlet characters to a large prime modulus $q$. More recently, Bombieri and Hejhal [1] have shown that Selberg's result for zeta is true for the values $\left\{L\left(\frac{1}{2}+i t\right)\right\}_{t \in[T, 2 T]}$ of a quite general $L$-function, under widely believed assumptions about the zeros of the function, and Wenzhi Luo 10 has verified this condition for the $L$-function associated to any fixed modular form for $S L_{2}(\mathbb{Z})$.

Following the ground-breaking work of Katz and Sarnak [6], we now understand the central values $L\left(\frac{1}{2}+i t\right)$ of an $L$-function as belonging in a family with a symmetry type governed by one of the classical compact groups. The cases considered thus far, of a fixed $L$-function with argument high in the critical strip, and of central values of Dirichlet $L$-functions with varying character of fixed conductor, arise as unitary families. On the basis of calculations from random matrix theory, Keating and Snaith [7] have proposed analogous Selberg-type conjectures for the logarithms of central values of $L$-functions from families of orthogonal and symplectic symmetry type, as well. These conjectures appear far from reach, however, because they involve only the real part of the logarithm of $L$-functions at the fixed point $s=\frac{1}{2}$; even the best known analytic methods have thus far only succeeded in proving that a positive proportion of $L$-functions in a family are non-zero at a single point, and even when the central value is known to be non-negative, the real part of the logarithm is highly sensitive to the 'low-lying' zeros, near $\frac{1}{2}$, which cannot presently be controlled. We will, however, prove partial results in two such cases. 
Let $S_{k}, k \equiv 0 \bmod 2$, be the space of weight $k$ modular cusp forms for $\mathrm{SL}_{2}(\mathbb{Z}) \backslash \mathbb{H}$ and let $H_{k}$ be its basis of $\sim \frac{k}{12}$ simultaneous eigenvectors of the Hecke operators, normalized to have first Fourier coefficient equal to 1 . Let $f \in H_{k}$ have Fourier expansion

$$
f(z)=\sum_{n=1}^{\infty} n^{\frac{k-1}{2}} \lambda_{f}(n) e(n z) .
$$

The $L$-function $L(s, f)$ associated to $f$ is then

$$
L(s, f)=\sum_{n=1}^{\infty} \frac{\lambda_{f}(n)}{n^{s}}=\prod_{p}\left(1-\frac{\lambda_{f}(p)}{p^{s}}+\frac{1}{p^{2 s}}\right)^{-1} .
$$

This has completed $L$-function

$$
\Lambda(s, f)=(2 \pi)^{-s} \Gamma\left(s+\frac{k-1}{2}\right) L(s, f),
$$

which satisfies the self-dual functional equation

$$
\Lambda(s, f)=i^{k} \Lambda(1-s, f) .
$$

When $k \equiv 2 \bmod 4$ this means that the central value $L\left(\frac{1}{2}, f\right)=0$, so for $k \equiv 0 \bmod 4$ we consider the family of values $\left\{L\left(\frac{1}{2}, f\right)\right\}_{f \in H_{k}}$, which is expected to have orthogonal symmetry type. These central values have a certain extra significance because Kohnen and Zagier proved the striking formula

$$
L\left(\frac{1}{2}, f\right)=\frac{c_{g}(1)^{2} \pi^{k}}{(k-1) !} \frac{\langle f, f\rangle}{\langle g, g\rangle}
$$

relating the central value $L\left(\frac{1}{2}, f\right)$ to the Petersson norm of $f$ and the first Fourier coefficient and Petersson norm of a half-integral weight form $g$ that lifts to $f$ under the Shimura correspondance. A particular consequence is that $L\left(\frac{1}{2}, f\right) \geq 0$; this is one of the few families of $L$-functions where non-negativity of the central value is known, although see [9] for a number of further examples.

As a second example we let $d>0$ be a fundamental discriminant with associated quadratic character $\chi_{d}(n)=\left(\frac{d}{n}\right)$ of conductor $d$. The corresponding Dirichlet $L$-function is

$$
L\left(s, \chi_{d}\right)=\sum_{n=1}^{\infty} \frac{\chi_{d}(n)}{n^{s}}=\prod_{p}\left(1-\frac{\chi_{d}(p)}{p^{s}}\right)^{-1}, \quad \Re(s)>1
$$

with completed $L$-function

$$
\Lambda\left(s, \chi_{d}\right)=\left(\frac{d}{\pi}\right)^{\frac{s+a}{2}} \Gamma\left(\frac{s+a}{2}\right) L\left(s, \chi_{d}\right), \quad a=\frac{1-\chi_{d}(-1)}{2} .
$$

This also satisfies the self-dual functional equation

$$
\Lambda\left(s, \chi_{d}\right)=\Lambda\left(1-s, \chi_{d}\right)
$$

and conjecturally $L\left(\frac{1}{2}, \chi_{d}\right) \geq 0$, but this is not known. For convenience we consider the family of central values $\left\{L\left(\frac{1}{2}, \chi_{8 d}\right)\right\}_{d \in s(D)}$ where $s(D)$ denotes the set of squarefree and odd $d, \frac{D}{2}<d \leq D$. In particular $\chi_{8 d}(-1)=1$ so that $a=0$ above. This is expected to be a family exhibiting symplectic symmetry. 
We have two primary results. The first result proves, unconditionally, 'one-half' of the Keating-Snaith conjectures.

Corollary 1.1. Let $k \equiv 0 \bmod 4$. As $k \rightarrow \infty$ we have

$$
\begin{array}{r}
\mathbb{P}\left[f \in H_{k}: \frac{1}{\sqrt{\log \log k}}\left(\log L\left(\frac{1}{2}, f\right)+\frac{1}{2} \log \log k\right)>A\right] \\
\leq \frac{1}{\sqrt{2 \pi}} \int_{A}^{\infty} e^{-\frac{x^{2}}{2}} d x+o_{A}(1) .
\end{array}
$$

In particular, for any fixed $\epsilon>0, L\left(\frac{1}{2}, f\right)<(\log k)^{-\frac{1}{2}+\epsilon}$ with probability $1-o_{\epsilon}(1)$. Also, as $D \rightarrow \infty$,

$$
\begin{aligned}
\mathbb{P}\left[d \in s(D): \frac{1}{\sqrt{\log \log D}}\left(\log \left|L\left(\frac{1}{2}, \chi_{8 d}\right)\right|\right.\right. & \left.\left.-\frac{1}{2} \log \log D\right)>A\right] \\
\leq & \frac{1}{\sqrt{2 \pi}} \int_{A}^{\infty} e^{-\frac{x^{2}}{2}} d x+o_{A}(1)
\end{aligned}
$$

In [15], Soundararajan made the basic observation that, on the Riemann Hypothesis, while zeros near $\frac{1}{2}+i t$ can greatly alter the value of $\log \left|\zeta\left(\frac{1}{2}+i t\right)\right|$, they tend to decrease its value as compared with that of $\log \left|\zeta\left(\frac{1}{2}+\sigma+i t\right)\right|$ at points off the critical line. Our proof of Corollary 1.1 is based upon an unconditional version of this fact, together with the following slightly technical result.

Theorem 1.2. Let $\sigma=\sigma(k)$ be a function of $k$, tending to 0 as $k \rightarrow \infty$ in such a way that $\sigma \log k \rightarrow \infty$ but $\frac{\sigma \log k}{\sqrt{\log \log k}} \rightarrow 0$. Also, for $f \in H_{k}$ put

$$
A(f)=\frac{1}{\sqrt{\log \log k}}\left(\log \left|L\left(\frac{1}{2}+\sigma, f\right)\right|+\frac{1}{2} \log \log k\right) .
$$

Then

$$
\frac{1}{\left|H_{k}\right|} \sum_{f \in H_{k}} \delta_{A(f)} \rightarrow N(0,1), \quad k \rightarrow \infty .
$$

Here $\delta_{x}$ is the point mass at $x, N(0,1)$ is the standard normal distribution, and the convergence is in the sense of distributions.

Similarly, let $\sigma=\sigma(D)$ be a function of $D$, tending to 0 as $D \rightarrow \infty$ in such a way that $\sigma \log D \rightarrow \infty$ but $\frac{\sigma \log D}{\sqrt{\log \log D}} \rightarrow 0$. For $d \in s(D)$, put

$$
A(d)=\frac{1}{\sqrt{\log \log D}}\left(\log \left|L\left(\frac{1}{2}+\sigma, \chi_{8 d}\right)\right|-\frac{1}{2} \log \log D\right) .
$$

Then

$$
\frac{1}{|s(D)|} \sum_{d \in s(D)} \delta_{A(d)} \rightarrow N(0,1), \quad D \rightarrow \infty
$$

This Theorem is proven using Selberg's method in [12]; in particular it makes use of 'zerodensity' estimates putting almost all of the low-lying zeros of the corresponding $L$-functions very near the half-line. In the case of $L\left(s, \chi_{8 d}\right)$, such a result is essentially available from the work of Conrey and Soundararajan in [2]. For the case of $L(s, f)$ see [4]. 
For our second main result we assume some weak conjectural information about the lowlying zeros in the families $\{L(s, f)\}_{f \in H_{k}}$, and $\left\{L\left(s, \chi_{8 d}\right)\right\}_{d \in s(D)}$ in order to deduce the full Keating-Snaith conjectures for these families. Given $f \in H_{k}$ and $s$ near $\frac{1}{2}, L(s, f)$ has conductor $\asymp k^{2}$, and therefore for $1 \ll T=k^{o(1)}$ the number of zeros of $L(s, f)$ up to height $T$ grows as $\frac{T}{\pi} \log k$. Reasoning probabilistically, we might then expect that for most $f \in H_{k}$, $\gamma_{\min }(f) \gg \frac{1}{\log k}$, where

$$
\gamma_{\min }(f)=\min _{\rho=\frac{1}{2}+\beta+i \gamma}|\gamma|
$$

is the height of the lowest non-trivial zero of $L(s, f)$. Similarly, for $d \in s(D)$ and $s$ near $\frac{1}{2}$, $L\left(s, \chi_{8 d}\right)$ has conductor $\asymp D$, and therefore we might typically expect that $\gamma_{\min }(d) \gg \frac{1}{\log D}$. We formalize this heuristic in the following hypothesis.

Hypothesis 1.3 (Low-lying Zero Hypothesis). Assume $y=y(k) \rightarrow \infty$ with $k$. Then

$$
\mathbb{P}\left[f \in H_{k}: \gamma_{\min }(f)<\frac{\pi}{y \log k}\right]=o(1), \quad k \rightarrow \infty .
$$

Similarly, if $y=y(D) \rightarrow \infty$ with $D$ then

$$
\mathbb{P}\left[d \in s(D): \gamma_{\min }(d)<\frac{2 \pi}{y \log D}\right]=o(1), \quad D \rightarrow \infty .
$$

In fact, stronger and more detailed statements about the low-lying zeros in these two families are expected to be true. Specifically, Iwaniec, Luo and Sarnak [5] have conjectured that in essentially any natural family of $L$-functions of conductor $C$, the one-level density of zeros at a scale of $\frac{2 \pi}{\log C}$ depends asymptotically only on the symmetry type of the family. For our two families of $L$-functions, their 'Zero Density Conjecture' takes the following shape.

Conjecture 1.4 (Zero Density Conjecture). Let $\phi(x)$ be a Schwarz class function on $\mathbb{R}$ with Fourier transform having compact support. Define the densities

$$
W\left(S O_{\text {even }}\right)(x) d x=\left(1+\frac{\sin 2 \pi x}{2 \pi x}\right) d x, \quad W(S p)(x) d x=\left(1-\frac{\sin 2 \pi x}{2 \pi x}\right) d x
$$

and write the non-trivial zeros of $L(s)$ as $\rho=\frac{1}{2}+i \gamma$, with $\gamma$ possibly complex if the Riemann Hypothesis for $L(s)$ is false. Then

$$
\lim _{\substack{k \rightarrow \infty \\
k \equiv 0 \bmod 4}} \frac{1}{\left|H_{k}\right|} \sum_{\substack { f \in H_{k} \\
\begin{subarray}{c}{\Lambda(\rho, f)=0 \\
\rho=\frac{1}{2}+i \gamma{ f \in H _ { k } \\
\begin{subarray} { c } { \Lambda ( \rho , f ) = 0 \\
\rho = \frac { 1 } { 2 } + i \gamma } }\end{subarray}} \phi\left(\frac{\gamma \log k}{\pi}\right)=\int_{-\infty}^{\infty} \phi(x) W\left(S O_{\text {even }}\right)(x) d x
$$

and

$$
\lim _{D \rightarrow \infty} \frac{1}{|s(D)|} \sum_{d \in s(D)} \sum_{\substack{\Lambda\left(\rho, \chi_{8 d}\right)=0 \\ \rho=\frac{1}{2}+i \gamma}} \phi\left(\frac{\gamma \log D}{2 \pi}\right)=\int_{-\infty}^{\infty} \phi(x) W(S p)(x) d x
$$

It is a straightforward exercise to prove that our Low-lying Zero Hypothesis is implied by the Zero Density Conjecture together with the Riemann Hypothesis for the corresponding family of $L$-functions.

We now state our second main result. 
Theorem 1.5. Suppose the Low-lying Zero Hypothesis holds for $\{L(s, f)\}_{f \in H_{k}}$. For $f \in H_{k}$ put

$$
B(f)=\frac{1}{\sqrt{\log \log k}}\left(\log L\left(\frac{1}{2}, f\right)+\frac{1}{2} \log \log k\right) .
$$

Then, as distributions

$$
\frac{1}{\left|H_{k}\right|} \sum_{f \in H_{k}} \delta_{B(f)} \rightarrow N(0,1), \quad k \rightarrow \infty
$$

Similarly, assume the Low-lying Zero Hypothesis for $\left\{L\left(s, \chi_{8 d}\right)\right\}_{d \in s(D)}$ and for $d \in s(D)$ put

$$
B(d)=\frac{1}{\sqrt{\log \log D}}\left(\log \left|L\left(\frac{1}{2}, \chi_{8 d}\right)\right|-\frac{1}{2} \log \log D\right) .
$$

Then, in the sense of distributions,

$$
\frac{1}{|s(D)|} \sum_{d \in s(D)} \delta_{B(d)} \rightarrow N(0,1), \quad D \rightarrow \infty
$$

In particular, either of these results is true if both the Riemann Hypothesis and the Zero Density Hypothesis is true for the corresponding family of L-functions.

\section{BACKGROUND}

In this section we collect together standard facts regarding our two families of $L$-functions, as well as the part of Selberg's work that we need for our arguments.

2.1. $L$-function coefficients, and orthogonality. For $f \in H_{k}$, the Fourier coefficients of $f$ satisfy the Hecke relations

$$
\lambda_{f}(m) \lambda_{f}(n)=\sum_{d \mid(m, n)} \lambda_{f}\left(\frac{m n}{d^{2}}\right) .
$$

A specific consequence of this fact is that for distinct primes $p_{1}, \ldots, p_{r}$ we have

$$
\lambda_{f}\left(p_{1}\right)^{e_{1}} \lambda_{f}\left(p_{2}\right)^{e_{2}} \cdots \lambda_{f}\left(p_{r}\right)^{e_{r}}=\sum_{0 \leq j_{1} \leq\left\lfloor\frac{e_{1}}{2}\right\rfloor} \cdots \sum_{0 \leq j_{r} \leq\left\lfloor\frac{e_{r}}{2}\right\rfloor} c(\mathbf{e}, \mathbf{j}) \lambda_{f}\left(p_{1}^{e_{1}-2 j_{1}} \cdots p_{r}^{e_{r}-2 j_{r}}\right)
$$

for some positive coefficients $c(\mathbf{e}, \mathbf{j})$.

Lemma 2.1. We have $c(\mathbf{2}, *)=1$ where $\mathbf{2}$ is the string consisting entirely of 2 's and $* i s$ any string containing only 0 's and 1's. Also, for general $\mathbf{e}, \mathbf{j}, c(\mathbf{e}, \mathbf{j}) \leq 2^{e_{1}+\ldots+e_{r}}$.

Recall, also, Deligne's bound $\left|\lambda_{f}(n)\right| \leq d(n)$.

We use the following basic orthogonality relation on $H_{k}$. 
Lemma 2.2. Let $0<\delta<2$. There exists $\gamma=\gamma(\delta)>0$ such that if $m<k^{2-\delta}$ then

$$
\frac{1}{\left|H_{k}\right|} \sum_{f \in H_{k}} \lambda_{f}(m)=\frac{\delta_{m=\square}}{\sqrt{m}}+O_{\delta}\left(k^{-\gamma}\right) \text {. }
$$

Proof. Actually, this is a combination of two different estimates. Using the Petersson Trace Formula, Rudnick and Soundararajan ([11], Lemma 2.1) prove that for $m n<\frac{k^{2}}{10000}$,

$$
\sum_{f \in H_{k}} \frac{2 \pi^{2}}{k-1} L\left(1, \operatorname{sym}^{2} f\right)^{-1} \lambda_{f}(m) \lambda_{f}(n)=\delta_{m=n}+O\left(e^{-k}\right) .
$$

Here $w_{f}=\frac{2 \pi^{2}}{k-1} L\left(1, \operatorname{sym}^{2} f\right)^{-1}$ is the 'harmonic weight' of $f$, and $L\left(s, \operatorname{sym}^{2} f\right)$ is the symmetric square $L$-function attached to $L(s, f)$ with coefficients given by

$$
L\left(s, \operatorname{sym}^{2} f\right)=\sum_{n=1}^{\infty} \frac{\rho_{f}(n)}{n^{s}}=\zeta(2 s) \sum_{n=1}^{\infty} \frac{\lambda_{f}\left(n^{2}\right)}{n^{s}}, \quad \Re(s)>1 .
$$

A now-standard method of Kowalski-Michel ([8, Proposition 2) allows the removal of the harmonic weight by truncating the Dirichlet series for $L\left(1, \operatorname{sym}^{2} f\right)$; with $x=\frac{k^{\delta / 2}}{100}$ and recalling $\left|H_{k}\right|=\frac{k-1}{12}+O(1)$, their method gives

$$
\begin{aligned}
\frac{1}{\left|H_{k}\right|} \sum_{f \in H_{k}} \lambda_{f}(m) & =\frac{1}{\left|H_{k}\right|} \sum_{f \in H_{k}} w_{f} \frac{k-1}{2 \pi^{2}} L\left(1, \operatorname{sym}^{2} f\right) \lambda_{f}(m) \\
& =\frac{1}{\zeta(2)} \sum_{f \in H_{k}} w_{f} \lambda_{f}(m) \sum_{\ell^{2} d<x} \frac{\lambda_{f}\left(d^{2}\right)}{\ell^{2} d}+O\left(k^{-\gamma}\right) .
\end{aligned}
$$

Substituting the bound of Rudnick and Soundararajan, one deduces the lemma.

For the real characters $\chi_{8 d}$, our basic orthogonality relation is the following.

Lemma 2.3. Let $n<D^{2-\delta}$. Then there is $\gamma=\gamma(\delta)>0$ such that

$$
\frac{1}{|s(D)|} \sum_{d \in s(D)}\left(\frac{8 d}{n}\right)=\delta_{n=\square} \prod_{\substack{p \mid n \\ \text { odd }}}\left(\frac{p}{p+1}\right)+O\left(D^{-\gamma}\right) .
$$

Proof. Note that $\mu(2 d)^{2}$ is the indicator function for odd, squarefree $d$. Rudnick and Soundararajan (11] Lemma 3.1) prove, for any $z>3$, that if $n$ is a perfect square then

$$
\sum_{d \leq z} \mu(2 d)^{2}\left(\frac{8 d}{n}\right)=\frac{z}{\zeta(2)} \prod_{p \mid 2 n}\left(\frac{p}{p+1}\right)+O\left(z^{\frac{1}{2}+\epsilon} n^{\epsilon}\right)
$$

and if $n$ is not a square then

$$
\sum_{d \leq z} \mu(2 d)^{2}\left(\frac{8 d}{n}\right)=O\left(z^{\frac{1}{2}} n^{\frac{1}{4}}\right) \log (2 n) .
$$

The result follows on taking successively $z=D / 2, D$. 
2.2. Selberg's work: two expressions for the logarithm. Writing the Euler product of $L(s, f)$ as

$$
L(s, f)=\prod_{p}\left(1-\frac{\lambda_{f}(p)}{p^{s}}+\frac{1}{p^{2 s}}\right)^{-1}=\prod_{p}\left(1-\frac{\alpha_{p}}{p^{s}}\right)^{-1}\left(1-\frac{\overline{\alpha_{p}}}{p^{s}}\right)^{-1}, \quad \Re(s)>1
$$

we have that for $m=1,2, \ldots$

$$
\lambda_{f}\left(p^{m}\right)=\alpha_{p}^{m}+\alpha_{p}^{m-2}+\ldots+\alpha_{p}^{-m+2}+\alpha_{p}^{-m}
$$

where for each $p, \alpha_{p}$ is a complex number of modulus 1 solving $\alpha_{p}+\overline{\alpha_{p}}=\lambda_{f}(p)$. Logarithmically differentiating $L(s, f)$ term-by-term we obtain

$$
-\frac{L^{\prime}}{L}(s, f)=\sum_{n=1}^{\infty} \frac{\Lambda_{f}(n)}{n^{s}}=\sum_{m=1}^{\infty} \sum_{p} \frac{\left(\alpha_{p}^{m}+\bar{\alpha}_{p}^{m}\right) \log p}{p^{m s}}, \quad \Re(s)>1 .
$$

In particular, $\Lambda_{f}(n)$ is supported on prime powers, and is given explicitly by

$$
\Lambda_{f}\left(p^{m}\right)=\left(\lambda_{f}\left(p^{m}\right)-\lambda_{f}\left(p^{m-2}\right)\right) \log p, \quad m \geq 1,
$$

with the convention that $\lambda_{f}\left(p^{-1}\right)=0$.

Similarly we have

$$
L\left(s, \chi_{8 d}\right)=\prod_{p}\left(1-\frac{\chi_{8 d}(p)}{p^{s}}\right)^{-1}, \quad \Re(s)>1
$$

and logarithmically differentiating this leads to

$$
-\frac{L^{\prime}}{L}\left(s, \chi_{8 d}\right)=\sum_{n} \frac{\Lambda_{8 d}(n)}{n^{s}}, \quad \Re(s)>1
$$

with $\Lambda_{8 d}$ supported on primes powers and

$$
\Lambda_{8 d}\left(p^{n}\right)=\left(\frac{8 d}{p^{n}}\right) \log p .
$$

In a standard way one may write down an expression for $-\frac{L^{\prime}}{L}(s, *)$ similar to (4) and (6) when $\frac{1}{2}<\Re(s) \leq 1$, although in this case the zeros of $L(s, *)$ enter into the formula. The following lemma is the analog of [13], Lemma 10 with $L(s, *)$ replacing the Riemann zeta function.

Lemma 2.4. Let $*$ stand in for either $8 d$ or $f$, so that $L(s, *)$ is either $L\left(s, \chi_{8 d}\right)$ for some $d \in s(D)$ or $L(s, f)$ for some $f \in H_{k}$.

Let $x>1$ be a parameter and define

$$
\Lambda_{x, *}(n)=\Lambda_{*}(n) a_{x}(n) ; \quad a_{x}(n)=\left\{\begin{array}{ll}
1, & 1 \leq n \leq x \\
\frac{\log ^{2} \frac{x^{3}}{n}-2 \log ^{2} \frac{x^{2}}{n}}{2 \log ^{2} x}, & x \leq n \leq x^{2} \\
\frac{\log ^{2} \frac{x^{3}}{n}}{2 \log ^{2} x}, & x^{2} \leq n \leq x^{3}
\end{array} .\right.
$$


For $s$ not coinciding with a trivial or non-trivial zero of $L(s, *)$ we have

$$
\begin{aligned}
-\frac{L^{\prime}}{L}\left(\frac{1}{2}+s, *\right)=\sum_{n \leq x^{3}} \frac{\Lambda_{x, *}(n)}{n^{\frac{1}{2}+s}} & +\frac{1}{\log ^{2} x} \sum_{\substack{\rho: \Lambda(\rho, *)=0 \\
\text { non-trivial }}} \frac{x^{\rho-\frac{1}{2}-s}\left(1-x^{\rho-\frac{1}{2}-s}\right)^{2}}{\left(\frac{1}{2}+s-\rho\right)^{3}} \\
& +\frac{1}{\log ^{2} x} \sum_{\substack{q: L(-q, *)=0, \Lambda(-q, *) \neq 0 \\
\text { trivial }}} \frac{x^{-q-\frac{1}{2}-s}\left(1-x^{-q-\frac{1}{2}-s}\right)^{2}}{\left(\frac{1}{2}+q+s\right)^{3}} .
\end{aligned}
$$

Proof. The sum $\sum_{n \leq x^{3}} \frac{\Lambda_{x, *}(n)}{n^{\frac{1}{2}+s}}$ is the result of expanding $-\frac{L^{\prime}}{L}(z, *)$ in its Dirichlet series in

$$
\frac{1}{2 \pi i \log ^{2} x} \int_{(3)} \frac{x^{z-\frac{1}{2}-s}\left(1-x^{z-\frac{1}{2}-s}\right)^{2}}{\left(z-\frac{1}{2}-s\right)^{3}}\left(-\frac{L^{\prime}}{L}(z, *)\right) d z
$$

and integrating term-by-term. The remainder of the expression is obtained by shifting the $z$-contour leftward and evaluating residues.

Introduce the gamma factors

$$
\gamma_{f}(s)=(2 \pi)^{-s} \Gamma\left(s+\frac{k-1}{2}\right), \quad \gamma_{8 d}(s)=\left(\frac{8 d}{\pi}\right)^{-\frac{s}{2}} \Gamma\left(\frac{s}{2}\right)
$$

so that we may write in a unified way

$$
\Lambda(s, *)=\gamma_{*}(s) L(s, *)
$$

for the completed $L$-function corresponding to either $L(s, f)$ or $L\left(s, \chi_{8 d}\right)$. The gamma factors do not play a significant role in our results; we only need

$$
\frac{\gamma_{f}^{\prime}}{\gamma_{f}}(\sigma)=\log k+O(1), \quad \frac{\gamma_{8 d}^{\prime}}{\gamma_{8 d}}(\sigma)=\frac{1}{2} \log D+O(1),
$$

uniformly in $\frac{1}{2} \leq \sigma \leq 1$. The completed $L$-function is entire of order 1 and hence has a Hadamard product running over its zeros,

$$
\Lambda(s, *)=e^{A+B s} \prod_{\rho: \Lambda(\rho, *)=0}\left(1-\frac{s}{\rho}\right) e^{\frac{s}{\rho}} .
$$

Logarithmically differentiating $\Lambda(s, *)$, and using that

$$
B=-\sum_{\rho: \Lambda(\rho, *)=0} \Re \frac{1}{\rho}
$$

as in [3], p. 82, one proves the following lemma.

Lemma 2.5. For real $\sigma>0$ we have

$$
-\frac{L^{\prime}}{L}\left(\frac{1}{2}+\sigma, *\right)=\frac{\gamma_{*}^{\prime}}{\gamma_{*}}\left(\frac{1}{2}+\sigma\right)-\sum_{\substack{\rho=\frac{1}{2}+\beta+i \gamma \\ \Lambda(\rho, *)=0}} \frac{\sigma-\beta}{(\sigma-\beta)^{2}+\gamma^{2}} .
$$


One of Selberg's major achievements in [13] was that he gave an efficient way to compute $\log \zeta\left(\frac{1}{2}+i t\right)$ as a short sum over primes. By balancing the expression for $-\frac{\zeta^{\prime}}{\zeta}(s)$ coming from the Hadamard product as in (12) against the expression from the Euler product (8), he was able to bound the contribution of the zeros in (8). To do so, Selberg introduced a perturbation $\sigma_{x, t}$ depending on the location of the zeros of $\zeta$ near height $t$, and evaluated $\log \zeta\left(\frac{1}{2}+\sigma_{x, t}+i t\right)$ in place of $\log \zeta\left(\frac{1}{2}+i t\right)$.

For $\log \left|L\left(\frac{1}{2}, *\right)\right|$ the analog of $\sigma_{x, t}$ is

$$
\begin{aligned}
& \sigma_{x, *}=2 \max _{\rho \in \mathcal{G}_{x, *}}\left(\beta, \frac{2}{\log x}\right) ; \\
& \mathcal{G}_{x, *}=\left\{\rho=\frac{1}{2}+\beta+i \gamma: \Lambda(\rho, *)=0,|\gamma| \leq \frac{x^{3|\beta|}}{\log x},|\beta| \geq \frac{2}{\log x}\right\} .
\end{aligned}
$$

Selberg's argument for $\log \zeta\left(\frac{1}{2}+\sigma_{x, t}+i t\right)$ carries over with trivial modifications to bound the zero sum of $L(s, *)$ at $s=\frac{1}{2}+\sigma_{x, *}$ and thus to the evaluation of $\log L\left(\frac{1}{2}+\sigma_{x, *}, *\right)$; the result is the following lemma.

Lemma 2.6. Let $C=k^{2}$ for $L(s, f)$ or $C=8 d$ for $L\left(s, \chi_{8 d}\right)$ be the conductor of the $L$ function near $s=\frac{1}{2}$. We have

$$
\sum_{\substack{\rho=\frac{1}{2}+\beta+i \gamma \\ \Lambda(\rho, *)=0}} \frac{\sigma_{x, *}}{\left(\sigma_{x, *}-\beta\right)^{2}+\gamma^{2}}=O\left(\left|\sum_{n<x^{3}} \frac{\Lambda_{x, *}(n)}{n^{\frac{1}{2}+\sigma_{x, *}}}\right|\right)+O(\log C)
$$

and

$$
\log L\left(\frac{1}{2}+\sigma_{x, *}, *\right)=\sum_{n \leq x^{3}} \frac{\Lambda_{x, *}(n)}{n^{\frac{1}{2}+\sigma_{x, *}} \log n}+O\left(\frac{1}{\log x}\left|\sum_{n \leq x^{3}} \frac{\Lambda_{x, *}(n)}{n^{\frac{1}{2}+\sigma_{x, *}}}\right|\right)+O\left(\frac{\log C}{\log x}\right) .
$$

Proof. See [13] pp 22-26.

In order to proceed further with Selberg's approach we need an understanding of the perturbation $\sigma_{x, *}$, that is, we need input regarding the distribution of zeros of $L(s, *)$ near the central point $s=\frac{1}{2}$ as either $f$ varies in $H_{k}$ or $d$ varies in $s(D)$. Our basic analytic ingredient is the following.

Theorem 2.7. For a sufficiently small $\delta>0$ there exists $\theta=\theta(\delta)$ such that, uniformly in $\frac{2}{\log k}<\sigma<\frac{1}{2}$ and $\frac{10}{\log k}<T<k^{2 \delta}$,

$$
\begin{aligned}
N(\sigma, T, k) & \stackrel{\text { def }}{=} \frac{1}{\left|H_{k}\right|} \sum_{f \in H_{k}} \#\left\{L\left(\frac{1}{2}+\beta+i \gamma, f\right)=0: \sigma<\beta,|\gamma|<T\right\} \\
& =O\left(T k^{-2 \theta \sigma} \log k\right)
\end{aligned}
$$


and also, uniformly in $\frac{4}{\log D}<\sigma<\frac{1}{2}$ and $\frac{10}{\log D}<T<D^{\delta}$,

$$
\begin{aligned}
N(\sigma, T, D) & \stackrel{\text { def }}{=} \frac{1}{|s(D)|} \sum_{d \in s(D)} \#\left\{L\left(\frac{1}{2}+\beta+i \gamma, \chi_{8 d}\right)=0: \sigma<\beta,|\gamma|<T\right\} \\
& =O\left(T D^{-\theta \sigma} \log D\right) .
\end{aligned}
$$

Proof. This result for $\{L(s, f)\}_{f \in H_{k}}$ is proved in [4].

The details of the second statement are largely contained in [2], but the situation is slightly different, so we briefly sketch the argument. The essential ingredient is an asymptotic evaluation of the twisted second moment

$$
\frac{1}{|s(D)|} \sum_{d \in s(D)}\left|L\left(\frac{1}{2}+\sigma+i t ; \chi_{8 d}\right)\right|^{2} \chi_{8 d}(\ell)=(\text { asymptotic main term })+O\left(D^{-\kappa} t^{A} \ell^{B}\right)
$$

uniform in the range $\sigma>0$ and $t<D^{\delta}$, where $\delta, \kappa$ and $A, B$ are fixed positive constants. The twisted second moment with power-saving error term was first obtained in this family at the central point $\sigma=t=0$ in [14], and in Propostion 2.3 of [2] the asymptotic is given for the range $0<\sigma=O(1),|t|=O(1)$ for the closely related family $\left\{L\left(s, \chi_{-8 d}\right)\right\}_{d \in s(D)}$. The extension of that result to the range $t=O\left(D^{\delta}\right)$ incurs no further difficulties; the limiting factor is the size of the analytic conductor $(|t|+D)$ of the family of $L$-functions, which in this case is essentially unchanged for $t$ as large as $D^{1-\epsilon}$. The authors in [2] also remark that their result remains valid in any arithmetic progression of fundamental discriminants, in particular, for the family $\left\{\chi_{8 d}\right\}_{d \in s(D)}$ considered here.

From an asymptotic formula for the twisted second moment it is a standard, albeit somewhat laborious, task to bound the mean-square

$$
\frac{1}{|s(D)|} \sum_{d \in s(D)}\left|\eta\left(\frac{1}{2}+\sigma+i t, \chi_{8 d}\right)\right|^{2} \leq 1+O\left(D^{-\theta \sigma}\right)+O\left(D^{-\frac{\kappa}{2}}|t|^{A}\right), \quad|t|<D^{\delta}
$$

where $\eta\left(s, \chi_{8 d}\right)=L\left(s, \chi_{8 d}\right) M\left(s, \chi_{8 d}\right)$ with $M\left(s, \chi_{8 d}\right)$ a short mollifying Dirichlet polynomial. The proof is then completed by appealing to a version of the argument principle to bound the total number of zeros of $\left\{\eta\left(s, \chi_{8 d}\right)\right\}_{d \in s(D)}$ in the specified box. This entire program is carried out for the family $\left\{L\left(s, \chi_{-8 d}\right)\right\}_{d \in s(D)}$ in the most difficult range where the box has height $T=O\left(\frac{1}{\log D}\right)$ in [2]. The method was first introduced in [12] and full details are contained there for the family of all Dirichlet $L$-functions to a fixed prime conductor. For another example calculation, see [4].

As a consequence we derive the following essential lemma.

Lemma 2.8. Let $\mathcal{F}$ be either the family of L-functions $\mathcal{F}=\{L(s, f)\}_{f \in H_{k}}$ or the family $\mathcal{F}=\left\{L\left(s, \chi_{8 d}\right)\right\}_{d \in s(D)}$. Denote $\mathbb{P}$ the uniform probability on $\mathcal{F}$. Let $C=k^{2}$ or $C=D$ be the respective conductor of the family.

For $x=x(C)$ growing with $C$ in such a way that $\frac{\log x}{\log C} \rightarrow 0$ as $C \rightarrow \infty$ we have

$$
\mathbb{P}\left[\exists \rho=\frac{1}{2}+\beta+i \gamma: \Lambda(\rho, *)=0, \beta>\frac{4}{\log x},|\gamma| \leq \frac{x^{3 \beta}}{\log x}\right]=o(1)
$$


as $C \rightarrow \infty$.

Proof. We may evidently assume that $\log x$ is larger than a fixed constant, and less than a sufficiently small constant times $\log C$. Then

$$
\begin{aligned}
\mathbb{P}\left[\exists \rho: \beta>\frac{4}{\log x},|\gamma| \leq \frac{x^{3 \beta}}{\log x}\right] & \leq \mathbb{P}\left[\bigcup_{j=4}^{\left\lceil\frac{\log x}{2}\right\rceil}\left\{\exists \rho: \beta>\frac{j}{\log x},|\gamma| \leq \frac{e^{3(j+1)}}{\log x}\right\}\right] \\
& \leq \sum_{j=4}^{\left\lceil\frac{\log x}{2}\right\rceil} \mathbb{P}\left[\exists \rho: \beta>\frac{j}{\log x},|\gamma| \leq \frac{e^{3(j+1)}}{\log x}\right] .
\end{aligned}
$$

By applying Theorem 2.7, the last sum is bounded by

$$
\ll \sum_{j=4}^{\left\lceil\frac{\log x}{2}\right\rceil} \frac{e^{3(j+1)}}{\log x} C^{-\frac{\theta j}{\log x}} \log C \leq \frac{\log C}{\log x} \sum_{j=4}^{\left\lceil\frac{\log x}{2}\right\rceil} e^{\left(-\theta \frac{\log C}{\log x}+3\right) j} \ll \frac{\log C}{\log x} e^{\frac{-\theta \log C}{\log x}}
$$

and this tends to zero as $C \rightarrow \infty$.

2.3. Convergence in the sense of distributions. Before turning to the main argument, we record, for repeated later use, the following simple fact concerning convergence in the sense of distributions.

Suppose we have a sequence of finite sets $\left\{R_{n}\right\}$. For each $n$ let there be two functions $f, \tilde{f}: R_{n} \rightarrow \mathbb{R}$, so that we obtain two sequences of probability measures $\left\{\mu_{n}\right\},\left\{\tilde{\mu}_{n}\right\}$ on $\mathbb{R}$,

$$
\mu_{n}=\frac{1}{\left|R_{n}\right|} \sum_{s \in R_{n}} \delta_{f(s)}, \quad \quad \tilde{\mu}_{n}=\frac{1}{\left|R_{n}\right|} \sum_{s \in R_{n}} \delta_{\tilde{f}(s)} .
$$

Lemma 2.9 (Distribution comparison lemma). Let $\mu$ be a finite (Borel) measure on $\mathbb{R}$. Each of the following three conditions is sufficient to guarantee the simultaneous convergence in distribution

$$
\mu_{n} \stackrel{d}{\longrightarrow} \mu \quad \Leftrightarrow \quad \tilde{\mu}_{n} \stackrel{d}{\longrightarrow} \mu
$$

of $\mu_{n}$ and $\tilde{\mu}_{n}$ to $\mu$.

$$
\begin{aligned}
\frac{1}{\left|R_{n}\right|} \sum_{\substack{s \in R_{n} \\
f(s) \neq \tilde{f}(s)}} 1 & =o(1), & n \rightarrow \infty \\
\sup _{s \in R_{n}}|f(s)-\tilde{f}(s)| & =o(1), & n \rightarrow \infty \\
\frac{1}{\left|R_{n}\right|} \sum_{s \in R_{n}}|f(s)-\tilde{f}(s)|^{2} & =o(1), & n \rightarrow \infty .
\end{aligned}
$$




\section{ThE Distribution OF THE PRIME SUM}

We first show that the short prime sums $\left(x=C^{o(1)}\right)$

$$
\left\{\sum_{n \leq x} \frac{\Lambda_{f}(n)}{\sqrt{n}}\right\}_{f \in H_{k}}, \quad\left\{\sum_{n \leq x} \frac{\Lambda_{8 d}(n)}{\sqrt{n}}\right\}_{d \in S(D)}
$$

converge to the appropriate Gaussian distributions as the conductor $C \rightarrow \infty$. The main work will then be in comparing $\log \left|L\left(\frac{1}{2}, *\right)\right|$ to these sums.

Proposition 3.1. Let $C=k^{2}$ for the family $\mathcal{F}=\{L(s, f)\}_{f \in H_{k}}$ and $C=D$ for the family $\left\{L\left(s, \chi_{8 d}\right)\right\}_{d \in s(D)}$. Assume $x=x(C)$ grows with $C$ in such a way that $\frac{\log x}{\log C} \rightarrow 0$ as $C \rightarrow \infty$, but $\log \log x=\log \log C+o(\sqrt{\log \log C})$. Define, for $f \in H_{k}$,

$$
P(f)=\frac{1}{\sqrt{\log \log k}}\left(\sum_{n \leq x} \frac{\Lambda_{f}(n)}{n^{\frac{1}{2}} \log n}+\frac{1}{2} \log \log k\right),
$$

and for $d \in s(D)$,

$$
P(d)=\frac{1}{\sqrt{\log \log D}}\left(\sum_{n \leq x} \frac{\Lambda_{8 d}(n)}{n^{\frac{1}{2}}}-\frac{1}{2} \log \log D\right) .
$$

We have

$$
\frac{1}{|\mathcal{F}|} \sum_{* \in \mathcal{F}} \delta_{P(*)} \stackrel{d}{\longrightarrow} N(0,1), \quad C \rightarrow \infty .
$$

Also, for each $C$ let $\left\{b_{n}(C)\right\}_{n=1}^{\infty}$ be a sequence of real numbers, bounded independently of $C$. Then

$$
\frac{1}{|\mathcal{F}|} \sum_{* \in \mathcal{F}}\left|\sum_{n<x^{3}} \frac{\Lambda_{*}(n) b_{n}}{n^{\frac{1}{2}}}\right|^{2}=O\left(\log ^{2} x\right), \quad C \rightarrow \infty .
$$

Proof. We show the proof for the family $\mathcal{F}=\{L(s, f)\}_{f \in H_{k}}$. The argument for real characters is essentially the same, with the caveat that the positive mean when the family is $\left\{L\left(s, \chi_{8 d}\right)\right\}_{d \in s(D)}$ comes from the fact that $\left(\frac{8 d}{p^{2}}\right)=1$ if $p \nmid 8 d$.

For (16), let $f \in H_{k}$ and write

$$
P(f)=\frac{1}{\sqrt{\log \log k}}\left[\sum_{m=1}^{\infty} \frac{1}{m} \sum_{p<x^{\frac{1}{m}}} \frac{\Lambda_{f}\left(p^{m}\right)}{p^{\frac{m}{2}} \log p}+\frac{1}{2} \log \log k\right] .
$$

Since $\Lambda_{f}\left(p^{m}\right)=\left(\lambda_{f}\left(p^{m}\right)-\lambda_{f}\left(p^{m-2}\right)\right) \log p$, with $\lambda_{f}\left(p^{-1}\right)=0$, we have

$$
\begin{aligned}
P(f) & =\frac{1}{\sqrt{\log \log k}} \sum_{p<x} \frac{\lambda_{f}(p)}{p^{\frac{1}{2}}}+\frac{1}{2 \sqrt{\log \log k}} \sum_{p<\sqrt{x}} \frac{\lambda_{f}\left(p^{2}\right)-1}{p}+\frac{1}{2} \sqrt{\log \log k}+o(1) \\
& =\frac{1}{\sqrt{\log \log k}} \sum_{p<x} \frac{\lambda_{f}(p)}{p^{\frac{1}{2}}}+\frac{1}{2 \sqrt{\log \log k}} \sum_{p<\sqrt{x}} \frac{\lambda_{f}\left(p^{2}\right)}{p}+o(1),
\end{aligned}
$$


by Mertens' theorem for $\sum \frac{1}{p}$. Regarding the second term, we may assume that $k$ is sufficiently large so that $x^{2}<k^{2-\delta}$. Then using orthogonality for $H_{k}$,

$$
\begin{aligned}
\frac{1}{\left|H_{k}\right|} \sum_{f \in H_{k}}\left[\sum_{p<\sqrt{x}} \frac{\lambda_{f}\left(p^{2}\right)}{p}\right]^{2} & =\frac{1}{\left|H_{k}\right|} \sum_{f \in H_{k}} \sum_{p<\sqrt{x}} \frac{1+\lambda_{f}\left(p^{2}\right)+\lambda_{f}\left(p^{4}\right)}{p^{2}}+\frac{1}{\left|H_{k}\right|} \sum_{f \in H_{k}} \sum_{p_{1} \neq p_{2}<\sqrt{x}} \frac{\lambda_{f}\left(p_{1}^{2} p_{2}^{2}\right)}{p_{1} p_{2}} \\
& =\sum_{p<\sqrt{x}}\left(\frac{1}{p^{2}}+\frac{1}{p^{3}}+\frac{1}{p^{4}}\right)+\sum_{p_{1} \neq p_{2} \leq x} \frac{1}{p_{1}^{2} p_{2}^{2}}+O\left(k^{-\gamma} \log ^{2} x\right)=O(1),
\end{aligned}
$$

so that, after normalizing by dividing by $\sqrt{\log \log k}$, this makes a negligible difference to the distribution ((iii) of Lemma 2.9). It thus suffices to demonstrate that for the prime sum

$$
\frac{1}{\left|H_{k}\right|} \sum_{f \in H_{k}} \delta_{\tilde{P}(f)} \stackrel{d}{\longrightarrow} N(0,1) ; \quad \tilde{P}(f)=\frac{1}{\sqrt{\log \log k}} \sum_{p<x} \frac{\lambda_{f}(p)}{p^{\frac{1}{2}}}
$$

This we do by the method of moments.

Let $m$ be fixed and assume now that $k$ is sufficiently large so that $x^{2 m}<k^{2-\delta}, x^{m}<k^{\frac{\gamma}{2}}$. We have

$$
\frac{1}{\left|H_{k}\right|} \sum_{f \in H_{k}}\left|\sum_{p<x} \frac{\lambda_{f}(p)}{p^{\frac{1}{2}}}\right|^{2 m}=\sum_{p_{1}, \ldots, p_{2 m}<x} \frac{1}{\sqrt{p_{1} \cdots p_{2 m}}} \frac{1}{\left|H_{k}\right|} \sum_{f \in H_{k}} \lambda_{f}\left(p_{1}\right) \cdots \lambda_{f}\left(p_{2 m}\right) .
$$

When some $p_{i}$ appears an odd number of times in the list, we see from the expression (3) that $\lambda_{f}\left(p_{1}\right) \cdots \lambda_{f}\left(p_{r}\right)$ can be written as a linear combination of $O_{m}(1)$ terms $\lambda_{f}\left(n_{i}\right)$, for which none of the $n_{i}$ are squares. Thus by Lemma 2.2 the contribution of all such terms is $\ll_{m} k^{-\frac{\gamma}{2}}$.

Among terms containing each $p_{i}$ an even number of times, those containing some $p_{i}$ at least 4 times contribute $\ll_{m}(\log \log x)^{m-2}$, which is an error term. We are left to consider terms containing each prime exactly twice. These contribute

$$
O_{m}\left(k^{-\gamma}(\log \log k)^{m}\right)+\sum_{\substack{p_{1}, \ldots, p_{m}<x \\ \text { distinct }}} \frac{1}{p_{1} \cdots p_{m}} \sum_{d \mid p_{1} \cdots p_{m}} \frac{1}{d}=\frac{(2 m) !}{2^{m} m !}(\log \log x)^{m}\left(1+o_{m}(1)\right)
$$

The claimed convergence in (18) thus follows from the fact that the Gaussian distribution is determined by its moments.

To prove (17), assume $x^{6}<\min \left(k^{2-\delta}, k^{\gamma}\right)$ and split the primes, prime squares, and higher powers as

$$
\left|\sum_{n<x^{3}} \frac{\Lambda_{f}(n) b_{n}}{n^{\frac{1}{2}}}\right|^{2} \leq 3\left[\left|\sum_{p<x^{3}} \frac{\lambda_{f}(p) b_{p} \log p}{p^{\frac{1}{2}}}\right|^{2}+\left|\sum_{p<x^{\frac{3}{2}}} \frac{O(\log p)}{p}\right|^{2}+O(1)\right]
$$


Thus

$$
\begin{aligned}
\frac{1}{\left|H_{k}\right|} \sum_{f \in H_{k}} & \left|\sum_{n<x^{3}} \frac{\Lambda_{f}(n) b_{n}}{n^{\frac{1}{2}}}\right|^{2} \leq \frac{3}{\left|H_{k}\right|} \sum_{f \in H_{k}}\left|\sum_{p<x^{3}} \frac{\lambda_{f}(p) b_{p} \log p}{p^{\frac{1}{2}}}\right|^{2}+O\left(\log ^{2} x\right) \\
& \leq 3 \sum_{p_{1}, p_{2} \leq x^{3}} \frac{b_{p_{1}} b_{p_{2}} \log p_{1} \log p_{2}}{\sqrt{p_{1} p_{2}}} \frac{1}{\left|H_{k}\right|} \sum_{f \in H_{k}} \lambda_{f}\left(p_{1}\right) \lambda_{f}\left(p_{2}\right)+O\left(\log ^{2} x\right) \\
& \leq \sum_{p \leq x^{3}} \frac{O\left(\log ^{2} p\right)}{p}+O\left(k^{-\gamma / 2}\right)+O\left(\log ^{2} x\right) \\
& =O\left(\log ^{2} x\right) .
\end{aligned}
$$

\section{Proof of MAIN RESUlts}

Throughout this section we let $\mathcal{F}$ be a family of $L$-functions, either $\mathcal{F}=\{L(s, f)\}_{f \in H_{k}}$ or $\mathcal{F}=\left\{L\left(s, \chi_{8 d}\right)\right\}_{d \in s(D)}$, and we let $C=k^{2}$ or $C=D$ for the conductor in the family. We also let $*$ stand in for the typical element in the family.

Proof of Theorem 1.2. Recall that in this theorem, $\sigma=\sigma(C)$ satisfies $\sigma \log C \rightarrow \infty$ while $\sigma \frac{\log C}{\sqrt{\log \log C}} \rightarrow 0$ as $C \rightarrow \infty$. Choose $x=x(C)$ by $\frac{4}{\log x}=\sigma$. Then we have $\log \log x=$ $\log \log C+O\left(\log _{3} C\right)$, so that we may appeal to Proposition 3.1 .

Recall that we set

$$
A(f)=\frac{1}{\sqrt{\log \log k}}\left(\log \left|L\left(\frac{1}{2}+\frac{4}{\log x}, f\right)\right|+\frac{1}{2} \log \log k\right)
$$

and

$$
A(d)=\frac{1}{\sqrt{\log \log D}}\left(\log \left|L\left(\frac{1}{2}+\frac{4}{\log x}, \chi_{8 d}\right)\right|-\frac{1}{2} \log \log D\right) .
$$

The theorem then asserts

$$
\frac{1}{|\mathcal{F}|} \sum_{* \in \mathcal{F}} \delta_{A(*)} \stackrel{d}{\longrightarrow} N(0,1)
$$

By Lemma 2.8 there is a set $E \subset \mathcal{F}$ of measure $o(1)$ such that outside $E, \sigma_{x, *}=\frac{4}{\log x}$. Thus by Selberg's approximation (15) we have

$$
\log L\left(\frac{1}{2}+\frac{4}{\log x}, *\right)=\sum_{n<x^{3}} \frac{\Lambda_{x, *}(n)}{n^{\frac{1}{2}+\frac{4}{\log x}} \log n}+O\left(\frac{1}{\log x}\left|\sum_{n \leq x^{3}} \frac{\Lambda_{x, *}(n)}{n^{\frac{1}{2}+\frac{4}{\log x}}}\right|\right)+O\left(\frac{\log C}{\log x}\right)
$$

on a set of measure $1-o(1)$. Note that the second error term contributes $o(1)$ to $A(*)$. Now

$$
\sum_{n<x^{3}} \frac{\Lambda_{x, *}(n)}{n^{\frac{1}{2}+\frac{4}{\log x}} \log n}=\sum_{n<x} \frac{\Lambda_{*}(n)}{n^{\frac{1}{2}} \log n}+\sum_{n<x} \frac{\Lambda_{*}(n)}{n^{\frac{1}{2}} \log n}\left(n^{\frac{-4}{\log x}}-1\right)+\sum_{x \leq n<x^{3}} \frac{\Lambda_{x, *}(n)}{n^{\frac{1}{2}+\frac{4}{\log x} \log n} .}
$$


The first term on the right is the prime sum, for which the convergence in distribution was proved in Proposition 3.1. Thus it will suffice to show that the first error term of (19) and the second and third terms above do not alter the distribution.

Applying (17) of Proposition 3.1 successively with corresponding choices of $b_{n}$, we find that

$$
\begin{aligned}
& \frac{1}{|\mathcal{F}|} \sum_{* \in \mathcal{F}}\left[\frac{1}{\log x} \sum_{n \leq x^{3}} \frac{\Lambda_{x, *}(n)}{n^{\frac{1}{2}+\frac{4}{\log x}}}\right]^{2}=O(1), \quad b_{n}=\frac{a_{x}(n)}{n^{\frac{4}{\log x}}} \\
& \frac{1}{|\mathcal{F}|} \sum_{* \in \mathcal{F}}\left[\sum_{n<x} \frac{\Lambda_{*}(n)}{n^{\frac{1}{2}} \log n}\left(n^{\frac{-4}{\log x}}-1\right)\right]^{2}=O(1), \quad b_{n}= \begin{cases}\frac{\log x}{\log n}\left(n^{\frac{-4}{\log x}}-1\right), & n \leq x \\
0, & x \leq n\end{cases} \\
& \frac{1}{|\mathcal{F}|} \sum_{* \in \mathcal{F}}\left[\sum_{x \leq n<x^{3}} \frac{\Lambda_{x, *}(n)}{n^{\frac{1}{2}+\frac{4}{\log x} \log n}}\right]^{2}=O(1), \quad b_{n}=\left\{\begin{array}{ll}
0, & n<x \\
\frac{a_{x}(n)}{n^{\frac{4}{\log x}} \frac{\log x}{\log n},} & x \leq n<x^{3}
\end{array} .\right.
\end{aligned}
$$

Thus by the distribution comparison lemma, Lemma 2.9,

$$
\frac{1}{|\mathcal{F}|} \sum_{* \in \mathcal{F}} \delta_{P(*)} \stackrel{d}{\longrightarrow} N(0,1) \quad \Rightarrow \quad \frac{1}{|\mathcal{F}|} \sum_{* \in \mathcal{F}} \delta_{A(*)} \stackrel{d}{\longrightarrow} N(0,1) .
$$

We will deduce Corollary 1.1 from Theorem 1.2 by comparing $\log \left|L\left(\frac{1}{2}, *\right)\right|$ and $\log \mid L\left(\frac{1}{2}+\right.$ $\left.\sigma_{x, *}, *\right) \mid$. Suppose that $L\left(\frac{1}{2}, *\right) \neq 0$. Then choosing a line integral that makes a small semicircle to avoid any real zero of $L(s, *)$,

$$
\begin{aligned}
\log \left|L\left(\frac{1}{2}, *\right)\right|- & \log L\left(\frac{1}{2}+\sigma_{x, *}, *\right)=\Re \int_{0}^{\sigma_{x, *}}-\frac{L^{\prime}}{L}\left(\frac{1}{2}+\sigma, *\right) d \sigma \\
= & \log \frac{\gamma_{*}\left(\frac{1}{2}+\sigma_{x, *}\right)}{\gamma_{*}\left(\frac{1}{2}\right)}-\sum_{\rho=\frac{1}{2}+\beta+i \gamma: \Lambda(\rho, *)=0} \Re \int_{0}^{\sigma_{x, *}} \frac{\sigma-\beta}{(\sigma-\beta)^{2}+\gamma^{2}} d \sigma \\
= & O(1)+\frac{1}{2} \sigma_{x, *} \log C-\frac{1}{2} \sum_{\rho=\frac{1}{2}+\beta+i \gamma: \Lambda(\rho, *)=0} \log \left(\frac{\left(\sigma_{x, *}-\beta\right)^{2}+\gamma^{2}}{\beta^{2}+\gamma^{2}}\right),
\end{aligned}
$$

as follows from the logarithmic derivative of the Hadamard product for $L(s, *)$ (12), and the approximation (10) to the logarithmic derivative of the gamma factor. When a zero $\rho$ is far to the right of the critical line, we pair the contribution of $\rho$ with that of its reflection $\rho^{\prime}$ in the line $\Re(s)=\frac{1}{2}$. Combined these contribute

$$
\begin{gathered}
\log \left[\frac{\left(\sigma_{x, *}-\beta\right)^{2}+\gamma^{2}}{\beta^{2}+\gamma^{2}} \cdot \frac{\left(\sigma_{x, *}+\beta\right)^{2}+\gamma^{2}}{\beta^{2}+\gamma^{2}}\right] \\
=\log \left[\left(1+\frac{\left(\sigma_{x, *}-2 \beta\right) \sigma_{x, *}}{\beta^{2}+\gamma^{2}}\right)\left(1+\frac{\left(\sigma_{x, *}+2 \beta\right) \sigma_{x, *}}{\beta^{2}+\gamma^{2}}\right)\right] \\
=\log \left[1+\frac{\sigma_{x, *}^{2}}{\beta^{2}+\gamma^{2}}\left(2+\frac{\sigma_{x, *}^{2}-4 \beta^{2}}{\beta^{2}+\gamma^{2}}\right)\right]
\end{gathered}
$$

to the sum over zeros in (20). 
Corollary 1.1 is now deduced by applying the following Proposition, which is an analog of the upper bound in the Proposition of [15], in the case when $\mathrm{RH}$ for the $L$-function is not assumed.

Proposition 4.1. Continue to let $\mathcal{F}=\{L(s, f)\}_{f \in H_{k}}$ or $\mathcal{F}=\left\{L\left(s, \chi_{8 d}\right)\right\}_{d \in s(D)}$, and let $C$ be the conductor of the L-functions in the family. For $\sigma_{x, *}$ as defined in (13) we have

$$
\log \left|L\left(\frac{1}{2}, *\right)\right| \leq \log L\left(\frac{1}{2}+\sigma_{x, *}, *\right)+\sigma_{x, *} \log C+O(1) .
$$

Proof. Since $L\left(\frac{1}{2}+\sigma_{x, *}, *\right) \neq 0$, if $L\left(\frac{1}{2}, *\right)=0$ then the claim holds, so we may suppose that $L\left(\frac{1}{2}, *\right) \neq 0$. For zeros $\rho=\frac{1}{2}+\beta+i \gamma$ such that $|\beta|<\frac{\sigma_{x, *}}{2}$, the contribution of $\beta$ to (20) is clearly negative, so we may assume $\beta>\frac{\sigma_{x, *}}{2}$ and pair $\rho$ and $\rho^{\prime}=\rho-2 \beta$. Since $\beta>\frac{\sigma_{x, *}}{2}$ we have $\rho \notin \mathcal{G}_{*}$ and thus

$$
\gamma>\frac{x^{3 \beta}}{\log x} \geq 3 \beta
$$

so that

$$
2 \geq 2+\frac{\sigma_{x, *}^{2}-4 \beta^{2}}{\beta^{2}+\gamma^{2}} \geq 2-\frac{4 \beta^{2}}{10 \beta^{2}} \geq \frac{8}{5}
$$

Hence

$$
\text { expr. (21) } \geq \log \left[1+\frac{8}{5} \frac{\sigma_{x, *}^{2}}{\beta^{2}+\gamma^{2}}\right]>0 .
$$

It follows that the paired zeros also contribute a negative amount to (20), which proves the Proposition.

Deduction of Corollary 1.1. Take $x=x(C)$ growing such that $\frac{\log C}{\log x} \rightarrow \infty$ but $\frac{\log C}{\log x \sqrt{\log \log C}} \rightarrow$ 0 as $C \rightarrow \infty$, as in the proof of Theorem 1.2. Then as before we have $\sigma_{x, *}=\frac{4}{\log x}$ except for on a set of measure $o(1)$. It follows from Proposition 4.1 that

$$
\frac{\log \left|L\left(\frac{1}{2}, *\right)\right|}{\sqrt{\log \log C}} \leq \frac{\log L\left(\frac{1}{2}+\frac{4}{\log x}, *\right)}{\sqrt{\log \log C}}+o(1)
$$

except on a set of measure $o(1)$, and the Corollary now follows from the convergence in distribution of the right hand side proved in Theorem 1.2 .

We now prove Theorem 1.5 by bounding the negative contribution of the zeros in (20) by invoking the Low-lying Zero Hypothesis.

Proof of Theorem 1.5. Let $x=x(C)$ and $y=y(C)$ be parameters growing with $C$, satisfying the conditions
(1) $\frac{\log C}{\log x} \rightarrow \infty$
(2) $\frac{\sqrt{\log \log C}}{\log y} \rightarrow \infty$, but $\frac{\log C}{y \log x} \rightarrow 0$
(3) $\frac{\log C \log y}{\log x \sqrt{\log \log C}} \rightarrow 0$ 
as $C \rightarrow \infty$. For instance, these are simultaneously satisfied with

$$
\log x=\log C(\log \log C)^{-\frac{1}{4}}, \quad y=\log \log C .
$$

Since $\frac{\log C}{\log x} \rightarrow \infty, \sigma_{x, *}=\frac{4}{\log x}$ except on a set of measure $o(1)$ in $\mathcal{F}$. Thus, invoking the Low-lying Zero Hypothesis, there is a subset $\mathcal{F}_{0} \subset \mathcal{F}$ of measure $1-o(1)$ in $\mathcal{F}$ which satisfies, for all $* \in \mathcal{F}_{0}, \sigma_{x, *}=\frac{4}{\log x}$ and for all zeros $\rho=\frac{1}{2}+\beta+i \gamma$ of $\Lambda(s, *)$,

$$
|\gamma|>\frac{1}{y \log x}
$$

Restricting to $\mathcal{F}_{0}$, by (20]) we have

$$
\begin{aligned}
& \log \left|L\left(\frac{1}{2}+\frac{4}{\log x}, *\right)\right|-\log \left|L\left(\frac{1}{2}, *\right)\right|= \\
& O\left(\frac{\log C}{\log x}\right)+\frac{1}{2} \sum_{\substack{\rho=\frac{1}{2}+\beta+i \gamma \\
\Lambda(\rho, *)=0}} \log \left(\frac{\left(\frac{4}{\log x}-\beta\right)^{2}+\gamma^{2}}{\beta^{2}+\gamma^{2}}\right) .
\end{aligned}
$$

In view of $\frac{\log C}{\log x}=o(\sqrt{\log \log C})$ this error term does not alter the distribution, so that, appealing to (iii) of Lemma 2.9 it suffices to prove the bound

$$
\frac{1}{|\mathcal{F}|} \sum_{* \in \mathcal{F}_{0}}\left|\sum_{\rho=\frac{1}{2}+\beta+i \gamma: \Lambda(\rho, *)=0} \log \left(\frac{\left(\frac{4}{\log x}-\beta\right)^{2}+\gamma^{2}}{\beta^{2}+\gamma^{2}}\right)\right|^{2}=o(\log \log C)
$$

in order to deduce the theorem from comparison with the normal approximation of $\log L\left(\frac{1}{2}+\right.$ $\left.\frac{4}{\log x}, *\right)$ proved in Theorem 1.2 .

In the sum over zeros of (†), for $\rho$ with $|\beta|<\frac{2}{\log x}$ we bound the summand in absolute value by

$$
\begin{aligned}
\left|\log \left(\frac{\left(\sigma_{x, *}-\beta\right)^{2}+\gamma^{2}}{\beta^{2}+\gamma^{2}}\right)\right| & =\left|\log \left(1-\frac{\left(\sigma_{x, *}-\beta\right)^{2}-\beta^{2}}{\left(\sigma_{x, *}-\beta\right)^{2}+\gamma^{2}}\right)\right| \\
& \leq\left|\log \left(1-\frac{\sigma_{x, *}^{2}}{\sigma_{x, *}^{2}+\gamma^{2}}\right)\right| \\
& =\left|\log \left(1-\frac{1}{1+\left(\frac{\gamma \log x}{4}\right)^{2}}\right)\right| \ll \frac{\log y}{1+\left(\frac{\gamma \log x}{4}\right)^{2}},
\end{aligned}
$$

by using $|\gamma| \log x \gg \frac{1}{y}$. Since $|\beta| \leq \frac{2}{\log x}$, the last quantity is bounded by

$$
\ll \frac{\log y}{\log ^{2} x} \frac{1}{\left(\frac{4}{\log x}-\beta\right)^{2}+\gamma^{2}} .
$$

For $|\beta|>\frac{2}{\log x}=\frac{\sigma_{x, *}}{2}$ we pair the contributions of $\rho$ and $\rho^{\prime}=\rho-2 \beta$. By (21) this combined contribution is

$$
\log \left[1+\frac{\sigma_{x, *}^{2}}{\beta^{2}+\gamma^{2}}\left(2+\frac{\sigma_{x, *}^{2}-4 \beta^{2}}{\beta^{2}+\gamma^{2}}\right)\right] .
$$


Since $|\beta|>\frac{\sigma_{x, *}}{2}$ we have $\rho, \rho^{\prime} \notin \mathcal{G}_{x, f}$, and therefore $|\gamma| \geq \frac{x^{3 \beta}}{\log x} \geq 3 \beta$. By (22),

$$
2 \geq 2+\frac{\sigma_{x, *}^{2}-4 \beta^{2}}{\beta^{2}+\gamma^{2}} \geq \frac{8}{5}
$$

and therefore the combined contribution is bounded in absolute value by

$$
\log \left[1+\frac{\sigma_{x, *}^{2}}{\beta^{2}+\gamma^{2}}\left(2+\frac{\sigma_{x, *}^{2}-4 \beta^{2}}{\beta^{2}+\gamma^{2}}\right)\right] \ll \frac{\sigma_{x, *}^{2}}{\beta^{2}+\gamma^{2}} \ll \frac{1}{\log ^{2} x} \frac{1}{\left(\frac{4}{\log x}-\beta\right)^{2}+\gamma^{2}} .
$$

Combining (23) and (24),

$$
\begin{aligned}
\left|\sum_{\rho=\frac{1}{2}+\beta+i \gamma} \log \left(\frac{\left(\frac{4}{\log x}-\beta\right)^{2}+\gamma^{2}}{\beta^{2}+\gamma^{2}}\right)\right| & =O\left(\frac{\log y}{\log ^{2} x} \sum_{\rho} \frac{1}{\left(\frac{4}{\log x}-\beta\right)^{2}+\gamma^{2}}\right) \\
& =O\left(\frac{\log y}{\log x} \mid \sum_{n \leq x^{3}} \frac{\Lambda_{x, *}(n)}{\left.n^{\frac{1}{2}+\frac{4}{\log x}} \mid\right)+O\left(\frac{\log y \log C}{\log x}\right)}\right.
\end{aligned}
$$

by Selberg's bound for sums over zeros in terms of sums over primes, (14) of Lemma 2.6.

Since

the bound (†) now follows from

$$
\frac{\log y \log C}{\log x}=o(\sqrt{\log \log C})
$$

$$
\frac{1}{|\mathcal{F}|} \sum_{* \in \mathcal{F}^{*}}\left|\frac{\log y}{\log x} \sum_{n \leq x^{3}} \frac{\Lambda_{x, *}(n)}{n^{\frac{1}{2}+\frac{4}{\log x}}}\right|^{2}=O\left(\log ^{2} y\right)=o(\log \log C)
$$

by (17) of Proposition 3.1.

\section{REFERENCES}

[1] E. Bombieri and D. A. Hejhal. On the distribution of zeros of linear combinations of Euler products. Duke Math. J., 80(3):821-862, 1995.

[2] J. B. Conrey and K. Soundararajan. Real zeros of quadratic Dirichlet L-functions. Invent. Math., 150(1):1-44, 2002.

[3] Harold Davenport. Multiplicative number theory, volume 74 of Graduate Texts in Mathematics. SpringerVerlag, New York, third edition, 2000. Revised and with a preface by Hugh L. Montgomery.

[4] Bob Hough. Zero density estimate for modular form $L$-functions in weight aspect. Acta Arith., 154:187216, 2012.

[5] Henryk Iwaniec, Wenzhi Luo, and Peter Sarnak. Low lying zeros of families of L-functions. Inst. Hautes Études Sci. Publ. Math., (91):55-131 (2001), 2000.

[6] Nicholas M. Katz and Peter Sarnak. Random matrices, Frobenius eigenvalues, and monodromy, volume 45 of American Mathematical Society Colloquium Publications. American Mathematical Society, Providence, RI, 1999.

[7] J. P. Keating and N. C. Snaith. Random matrix theory and L-functions at $s=1 / 2$. Comm. Math. Phys., 214(1):91-110, 2000.

[8] E. Kowalski and P. Michel. The analytic rank of $J_{0}(q)$ and zeros of automorphic $L$-functions. Duke Math. J., 100(3):503-542, 1999.

[9] Erez M. Lapid. On the nonnegativity of Rankin-Selberg $L$-functions at the center of symmetry. Int. Math. Res. Not., (2):65-75, 2003. 
[10] Wen Zhi Luo. Zeros of Hecke L-functions associated with cusp forms. Acta Arith., 71(2):139-158, 1995.

[11] Z. Rudnick and K. Soundararajan. Lower bounds for moments of $L$-functions: symplectic and orthogonal examples. In Multiple Dirichlet series, automorphic forms, and analytic number theory, volume 75 of Proc. Sympos. Pure Math., pages 293-303. Amer. Math. Soc., Providence, RI, 2006.

[12] Atle Selberg. Contributions to the theory of Dirichlet's L-functions. Skr. Norske Vid. Akad. Oslo. I., 1946(3):62, 1946.

[13] Atle Selberg. Contributions to the theory of the Riemann zeta-function. Arch. Math. Naturvid., 48(5):89$155,1946$.

[14] K. Soundararajan. Nonvanishing of quadratic Dirichlet $L$-functions at $s=\frac{1}{2}$. Ann. of Math. (2), 152(2):447-488, 2000.

[15] Kannan Soundararajan. Moments of the Riemann zeta function. Ann. of Math. (2), 170(2):981-993, 2009.

E-mail address: rdhough@gmail.com 